\title{
Artificial insect wings of diverse morphology for flapping-wing micro air vehicles
}

\section{Citation}

Shang, J K, S A Combes, B M Finio, and R J Wood. 2009. "Artificial Insect Wings of Diverse Morphology for Flapping-Wing Micro Air Vehicles." Bioinspiration \& Biomimetics 4 (3) (August 27): 036002. doi:10.1088/1748-3182/4/3/036002.

\section{Published Version}

doi:10.1088/1748-3182/4/3/036002

\section{Permanent link}

http://nrs.harvard.edu/urn-3:HUL.InstRepos:25283810

\section{Terms of Use}

This article was downloaded from Harvard University's DASH repository, and is made available under the terms and conditions applicable to Open Access Policy Articles, as set forth at http:// nrs.harvard.edu/urn-3:HUL.InstRepos:dash.current.terms-of-use\#OAP

\section{Share Your Story}

The Harvard community has made this article openly available.

Please share how this access benefits you. Submit a story.

Accessibility 


\title{
Artificial insect wings of diverse morphology for flapping-wing MAVs
}

\author{
J. K. Shang ${ }^{1}$, S. A. Combes ${ }^{2}$, B. M. Finio ${ }^{1}$, and R. J. Wood ${ }^{1,3}$ \\ ${ }^{1}$ School of Engineering and Applied Sciences, Harvard University, Cambridge, MA \\ 02138 \\ ${ }^{2}$ Dept. of Organismic and Evolutionary Biology, Harvard University, Cambridge, \\ MA 02138 \\ E-mail: ${ }^{3}$ rjwood@seas.harvard.edu
}

\begin{abstract}
The development of flapping-wing micro air vehicles (MAVs) demands a systematic exploration of the available design space to identify ways in which the unsteady mechanisms governing flapping-wing flight can best be utilized for producing optimal thrust or maneuverability. Mimicking the wing kinematics of biological flight requires examining the potential effects of wing morphology on flight performance, as wings may be specially adapted for flapping flight. For example, insect wings passively deform during flight, leading to instantaneous and potentially unpredictable changes in aerodynamic behavior. Previous studies have postulated various explanations for insect wing complexity, but there lacks a systematic approach for experimentally examining the functional significance of components of wing morphology, and for determining whether or not natural design principles can or should be used for MAVs. In this work, a novel fabrication process to create centimeter-scale wings of great complexity is introduced; via this process, a wing can be fabricated with a large range of desired mechanical and geometric characteristics. We demonstrate the versatility of the process through the creation of planar, insect-like wings with biomimetic venation patterns that approximate the mechanical properties of their natural counterparts under static loads. This process will provide a platform for studies investigating the effects of wing morphology on flight dynamics, which may lead to the design of highly maneuverable and efficient MAVs and insight into the functional morphology of natural wings.
\end{abstract}


Key index words: Insect aerodynamics, micro air vehicles, flapping-wing aerodynamics, biomimetics, wing flexibility

\section{Introduction}

In recent years, interest has surged in small-scale autonomous or remotely controlled aircraft - Micro Air Vehicles (MAVs) - with broad applications in hazardous environment exploration, reconnaissance, and search and rescue. Small-scale MAVs aspire to high levels of maneuverability and aerodynamic efficiency; since remarkable flight performance is achieved by birds, bats, and flying insects at the desired scale, research efforts have typically focused on a biologically-inspired approach to MAV design (e.g. $[1,2,3,4,5])$.

Within the biologically-inspired design paradigm, much attention has been given to the challenge of understanding the unsteady mechanisms that govern flapping flight. In the present work, we focus on insect flight, as insects are at the desired scale and exhibit simpler control processes than birds and bats, which possess active musculature along the span of the wing [6]. Though most studies consider wingbeat kinematics critical to lift generation [7], few address the shape and mechanical properties of the wings themselves [22, 23]. Unlike aircraft wings, biological wings are textured, flexible, and thin; wings passively bend and twist appreciably in flight, leading to instantaneous changes in aerodynamic behavior, as wing shape and fluid forces are dynamically coupled. It has been suggested that deformation increases lift production by directing fluid forces in desired directions [8, 9]. Flexible airfoils for MAVs appear to delay stall at higher angles of attack [29, 30]. Hence biologically inspired wings may have advantages for flapping MAVs.

Drawing upon these advantages first requires an understanding of the effects of wing morphology. Naturally occurring wings vary widely in venation, cross-sectional, and planform geometries, but methods of evaluating the functional consequences of wing morphology are limited. Detailed measurements of the forces produced by live insects during flight are not possible with current technology. Dynamically scaled physical models can provide valuable information about the effects of kinematic changes on force production [7], but wing flexibility cannot be scaled appropriately in these models. Numerical models of flapping wings are computationally expensive, and even under static loads, often fail to quantitatively match empirical behavior $[27,28,25]$.

Instead, effects of particular morphological features can be rapidly tested by creating artificial, at-scale wings and subjecting them to static or dynamic loads; unconstrained by natural design space, an artificial wing's morphological and material parameters can be carefully manipulated, and their effects on force production observed. Wing fabrication methods for flapping MAVs exist, but are scarce. Existing methods are capable of achieving varying levels of structural complexity 
and mechanical stiffness, but often are geometrically limited or sacrifice mechanical anisotropy - a trait of biological wings - via vein material choice [31, 32, 4]. This work introduces a novel fabrication process capable of achieving both high structural complexity and realistic mechanical stiffness, inspired by the following morphological considerations:

- Planform geometry. The magnitude of induced drag from tip vortices varies inversely with a wing's aspect ratio. Hence high aspect ratio wings should be more efficient than low aspect ratio wings, yet most insects do not have high aspect ratio wings [6]. Long and slender wings may simply be impractical for insects that fold their wings, or there may be a compromise between aspect ratio, kinematics, and power requirements. For a steadily translating wing, maximizing aspect ratio is optimal, yet for oscillating wings a high aspect ratio increases the moment of inertia, increasing power requirements and potentially changing resonance and wingbeat kinematics. Within wing planforms of similar aspect ratio, shape distribution results in variable performance, and local optima in wingbeat frequency and shape distribution seem to exist $[13,14]$.

- Venation. Local variation in material properties is augmented by the pattern of supporting veins, which ranges from extremely complex to highly reduced among insect orders [21]. Shape and size vary among and within veins; vein stiffness tends to decrease spanwise towards the tip and chordwise to the trailing edge, making the wing more flexible to impact while reducing its moment of inertia [19]. Consequently, the wing possesses local regions that are especially deformable or rigid, which contribute to patterns of bending and torsion in flight. Though flexural stiffness seems to be dominated by the stiffness at the leading edge and near the wing base, local stiffness may have a significant effect on the distribution of dynamic pressure stresses [22, 23].

The fabrication process described here is capable of producing lightweight, complex, centimeter-scale artificial wings, permitting customizable variations in planform geometry and venation structure and thus enabling experiments to systematically evaluate the effects of each parameter. The process achieves both the structural complexity and mechanical stiffness sought by other processes via the use of unidirectional carbon fiber veins - where the high elastic modulus helps to match the stiffness of natural, hollow veins [21]. In this paper we briefly illustrate the process's potential to create planar wings with complex venation patterns that, when statically loaded, are comparable to natural wings to a first order. Though dynamic loading is critical to aerodynamic performance, at this time suitable methods of experimentally measuring dynamic pressures and/or forces across a flapping insect-sized wing are unknown, and hence the current work relies on static tests similar to those that have been performed on real insect wings [22]. 


\section{Materials and methods}

Artificial wings were created with venation patterns derived from a selection of wings used by Combes and Daniel in their flexural stiffness study [22]. Because we sought to simply demonstrate the feasibility of the process and approximate mechanical properties, planar wings (without three-dimensional relief) were created as a first step, and some designs were simplified. Extreme curvatures were reduced (as discussed later), complex structures (e.g. cellular venation in Odonata) were removed, and all veins within a wing were created to uniform width. A planar film of uniform thickness served as the membrane.

The fabrication process used to create the wings is a combination of Smart Composite Microstructures (SCM) and soft lithography [33, 34]. Poly(dimethysiloxane) is cast onto a master having the relief structure of the desired vein design, where the height of the relief structure is the desired vein height (Fig. 1.3). Many techniques are available to create this master (e.g. photolithography, Figs. 1.1, 1.2). After curing, the elastomer is peeled off and silanized (Fig. 1.4), producing a wing mold.

A biomimetic wing must be extremely lightweight and requires a flexible membrane reinforced by a framework of stiff, lightweight veins. Emulating the material choice of the Harvard University microrobotic fly, veins laser-micromachined from unidirectional carbon fiber prepreg $[2] \ddagger$ - chosen for its high elastic modulus are inlaid into the mold's channels (Fig. 1.5), facilitated by the anisotropic properties of the material. A membrane of polymer thin film§ is aligned on top (Fig. 1.6). We expect that the high elastic modulus of the carbon fiber ( $\sim 300 \mathrm{GPa})$ will dominate the wing's structural properties.

The assembly is then cured by vacuum bagging to ensure uniform pressure distribution during the cure cycle. The cured wing is released from the substrate (Fig. 1.7), and the superfluous venation is removed (Fig. 1.8). Steps 5-8 may be iteratively repeated to yield a wing of greater complexity or non-homogeneous vein geometry (e.g. additional height to individual veins).

The achievable venation curvature is constrained by the mechanical properties of the carbon fiber. If a curved vein can be spatially described as the deflection of a beam $y(x)$ from an unloaded state, where $x$ is the position along the beam (Fig. 2 ), then the upper bound of curvature at position $x_{0}$ is governed by Euler-Bernoulli beam theory, where $y$ must satisfy the condition

$$
\sigma=E h \frac{d^{2} y}{d x^{2}}<\sigma_{\max }
$$

evaluated at $x_{0}$, where $E$ is Young's modulus, $h$ is the maximum height from the neutral axis, and $\sigma_{\max }$ is the tensile strength. This is an upper bound because some of the stress will inevitably be distributed to the surrounding elastomeric matrix.

$\ddagger$ M60J or M55J fibers (Toray America) pre-impregnated with resin RS-3C (YLA Inc.)

$\S 1.5 \mu \mathrm{m}$ ultra polyester thin film (Chemplex Industries) 


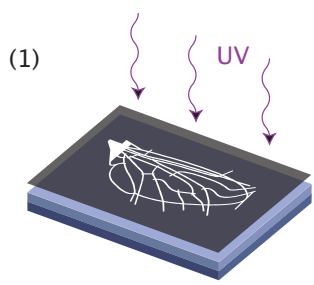

(5)

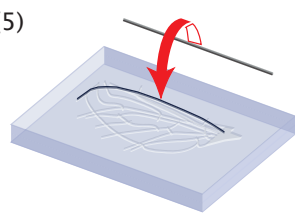

(2)

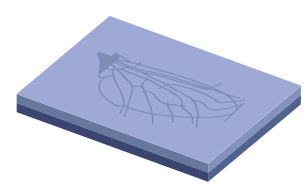

(6)

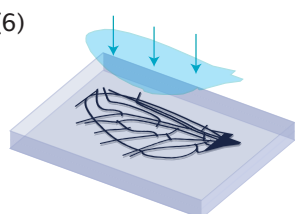

(3)

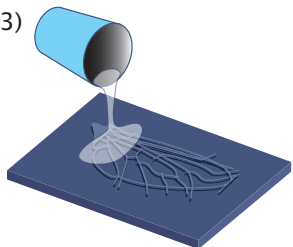

(7)

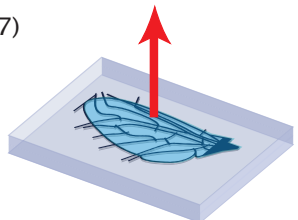

(4)

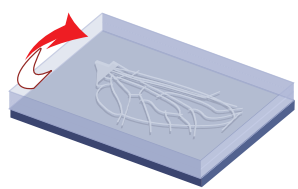

(8)

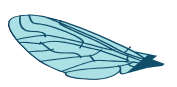

Figure 1. Wing fabrication process, using photolithography to create a positive relief. (1) A transparency mask of the wing is overlaid onto a Si wafer spincoated with photoresist, and exposed to UV light. (2) The unexposed photoresist is removed with a solvent, and the wafer is silanized. (3) PDMS is poured over the relief and cured. (4) The PDMS mold is removed from the wafer and silanized. (5) Laser-micromachined carbon fiber prepreg veins are inlaid into the mold channels. (6) A membrane is laid on top, and the assembly is cured via a vacuum-bagging process. (7) The wing is released from the mold, and (8) the superfluous venation removed.

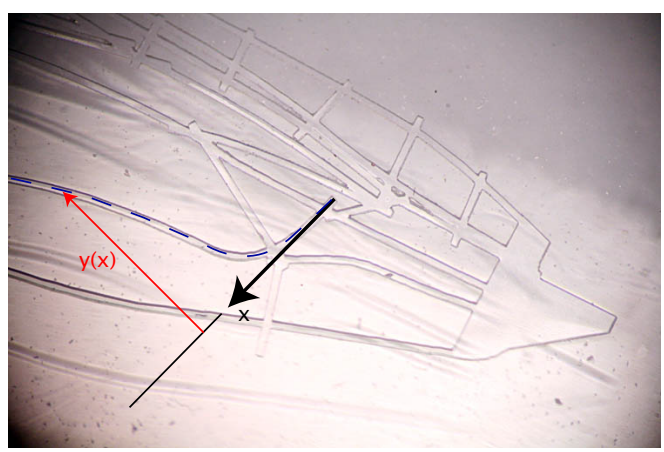

Figure 2. Coordinate system for determining the possible deflection of a vein.

\section{Discussion}

Examples of artificial wings are shown in Fig. 3. To verify that wings made with this process have mechanical properties comparable to real wings, the artificial wings were subjected to static loading tests to determine the overall flexural stiffness, EI, in the chordwise and spanwise directions. Though the wings clearly are not homogenous beams, calculating the overall flexural stiffness provides a simple measure with which to compare to existing data on natural wings [22]. Suitable methods of dynamic 
Table 1. Manipulation of stiffness properties via changes in vein geometry

\begin{tabular}{|r||c|c|}
\hline & Spanwise $E I\left(\mathrm{~N}-m^{2}\right)$ & Chordwise $E I\left(\mathrm{~N}-m^{2}\right)$ \\
\hline Initial biomimetic wing & $5.1 \times 10^{-6} \pm 2.9 \times 10^{-8}$ & $2.1 \times 10^{-7} \pm 4.8 \times 10^{-9}$ \\
\hline Wing with increased vein height & $6.6 \times 10^{-6} \pm 4.2 \times 10^{-8}$ & $3.0 \times 10^{-7} \pm 5.9 \times 10^{-9}$ \\
\hline
\end{tabular}

testing, which may provide more information about wing deformation patterns during flight, have not been developed for real wings and so are not employed here.

The wings were glued to glass slides with cyanoacrylate glue at the wing base (for spanwise measurements) or the leading edge (for chordwise measurements). The slide was lowered by a micrometer stage until it came into contact with a pin mounted on a balance (Fig. 4). Lowering the slide further applied a point load to the wing; the deflection was recorded from the micrometer $(\delta)$ and the applied force from the balance $(F)$. The flexural stiffness $E I$ was calculated as

$$
E I=F L^{3} / 3 \delta
$$

where $L$ is the distance between wing attachment and the location of the point load, which was approximately $70 \%$ span or chord. This distance was chosen to be consistent with the experimental setup executed by Combes and Daniel [22]. Deflections were less than or equal to $5 \%$ of the beam length $L$ because Eq. (2) is only applicable for small deflections.

Measurements of spanwise and chordwise stiffness compare well with natural wings of equivalent span or chord, falling on the same power-law relationship (Fig. 5 top and center). Wings also demonstrate similar spanwise-chordwise anisotropy, with chordwise EI being 1-2 orders of magnitude smaller than spanwise EI (Fig. 5 bottom). Wings previously used in the Harvard microrobotic fly [2] are substantially less stiff than natural wings or wings fabricated with the new process.

The mechanical properties can be easily manipulated by adjusting the geometry or material of a wing. One venation pattern was replicated with a $25 \%$ increase in vein height, yielding increases in $E I$ in both the spanwise and chordwise directions (Table 1), of $28 \%$ and $41 \%$, respectively.

\section{Conclusions}

The fabrication method outlined here provides a starting point for experiments investigating the aerodynamic performance of wings with a wide array of user-defined mechanical properties. As illustrative examples, the artificial wings manufactured here intentionally have static properties comparable to natural wings to a first 


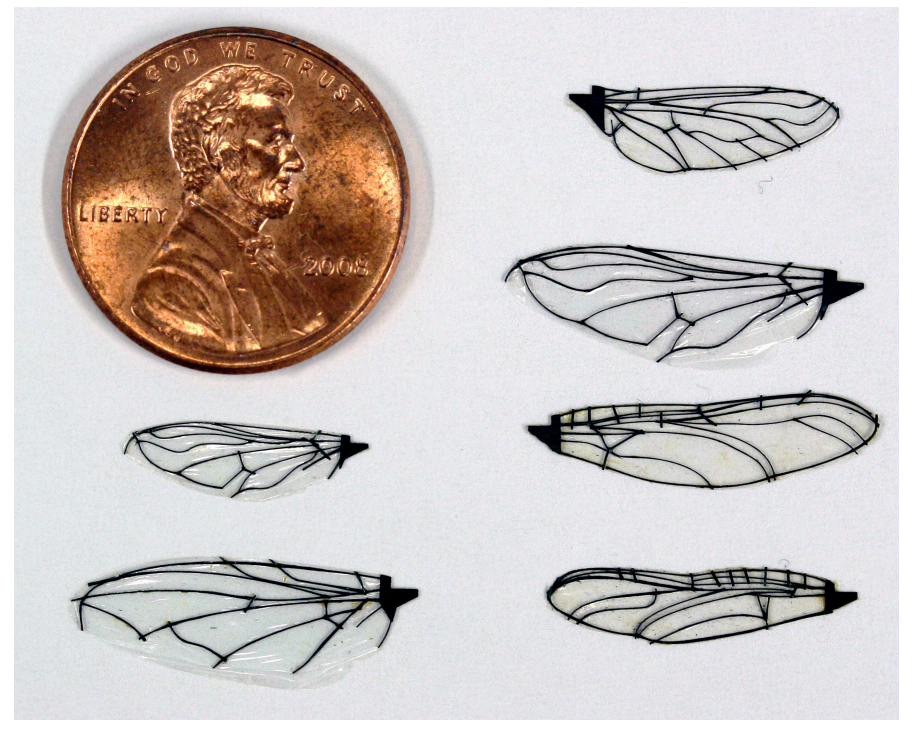

Figure 3. Examples of biomimetic wings used for mechanical testing.

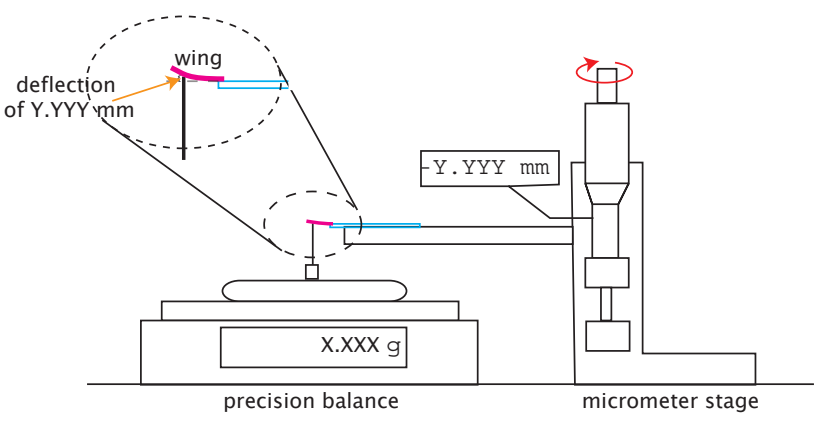

Figure 4. Experimental setup. A wing mounted on a glass slide is deflected a known amount by applying a point load on the wing surface.

approximation, though wings created with this process are by no means constrained to such a range.

Though we have created planar wings here, previous airfoil and insect studies indicate that $3 \mathrm{D}$ wing relief (e.g. camber or corrugation) may be crucial to force production, as well as to determining patterns of dynamic bending and torsion. A steadily translating wing experiences higher lift-to-drag ratios with decreasing thickness and increasing camber [15]. Because wings often passively deform to eliminate or reverse camber on the upstroke, one should expect instantaneous changes in aerodynamic properties as well [16]. Camber also influences torsional rigidity, producing asymmetric twisting behavior between half-strokes, as noted in butterflies [17]. Changes in shape due to bending and twisting further affect torsional and bending behavior [18]. Wing corrugation as seen in dragonflies (Odonata) has been 

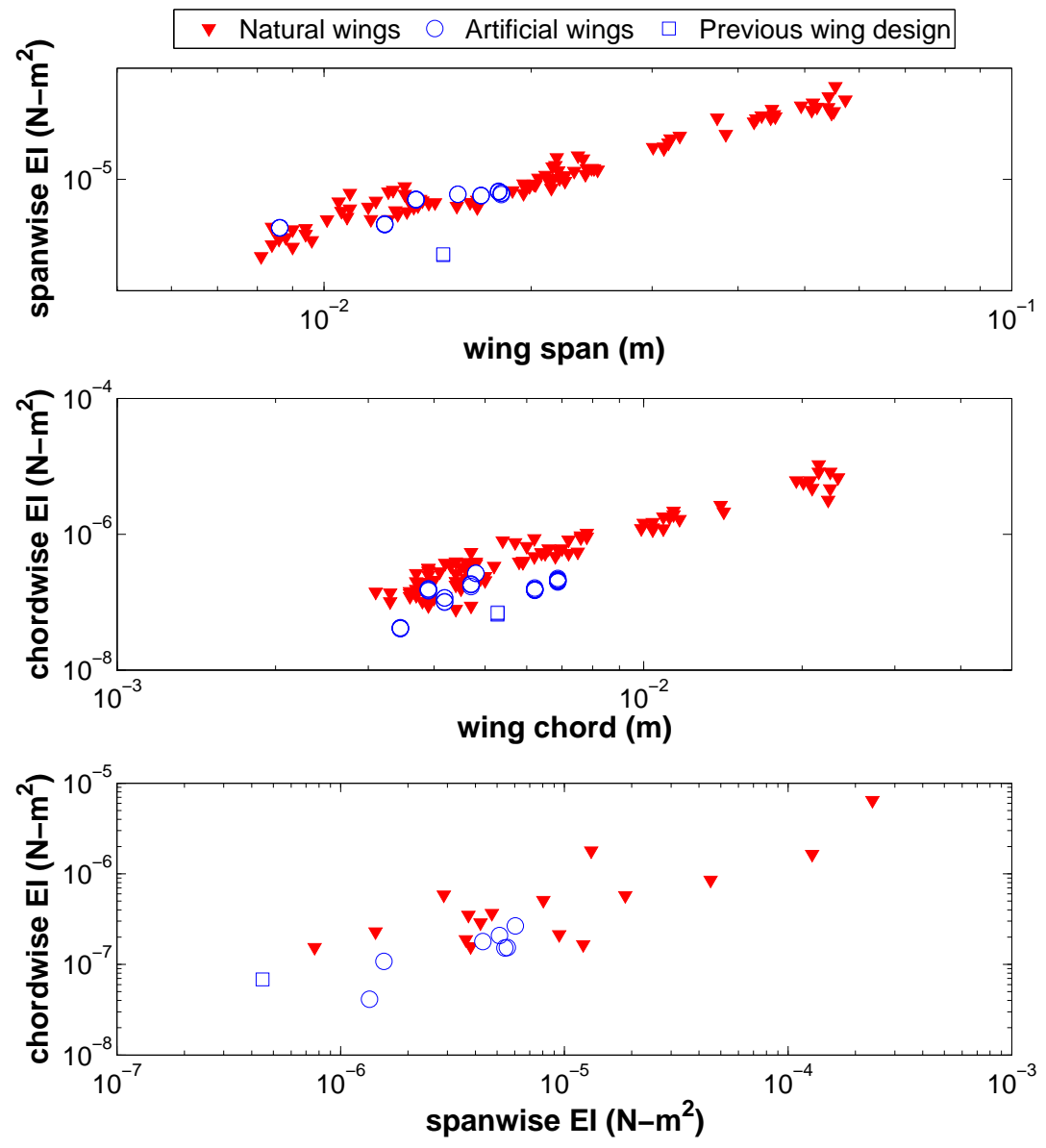

Figure 5. Biomimetic wings compared with data from real wings [22]: flexural stiffness versus span length (top); flexural stiffness versus chord length (center); anisotropic flexural stiffness in the chordwise and spanwise directions (bottom).

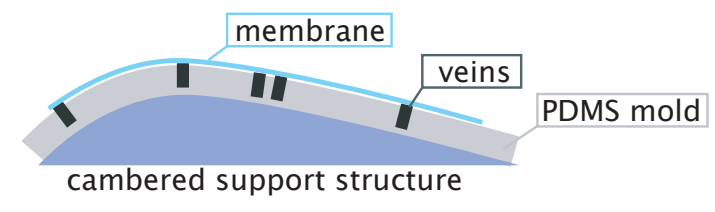

(a)

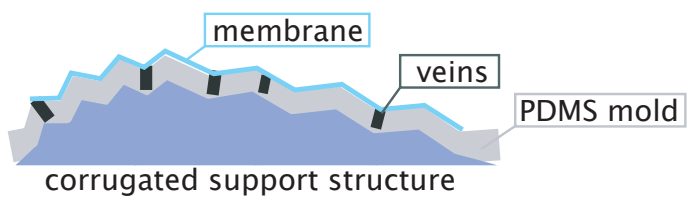

(b)

Figure 6. Possible fabrication of cambered and/or corrugated wings. 
shown to affect near-field flow and thus may be aerodynamically beneficial [15, 20], while also resisting spanwise bending [19]. The process described here can be modified to accommodate camber or corrugated profiles by fitting the PDMS mold into the desired 3D shape and curing the wing in that configuration (Figs. 6(a), 6(b)). In addition, spin-coating (or other means of depositing) the polymer membrane onto the venation pattern would allow greater freedom in cross-sectional profile geometry. Through an appropriate choice of polymer for the membrane, an etching process could further manipulate the spatial distribution of stiffness, possibly introducing highly flexible fold and flexion lines as on biological wings [21].

The question of whether or not artificial wings created by this process will demonstrate dynamic bending behavior that is also similar to natural wings - and thus would exploit any aerodynamic benefit that insects may derive from wing deformation - is beyond the scope of this work. The distribution of wing mass, as well as stiffness, will affect dynamic bending, and this parameter has not yet been tested in the artificial wings. However, because mass distribution is correlated with venation pattern in real wings [6], it is probable that artificial wings with biologically-inspired venation patterns could be optimized to exhibit dynamic deflections similar to those found in nature.

\section{Future Work}

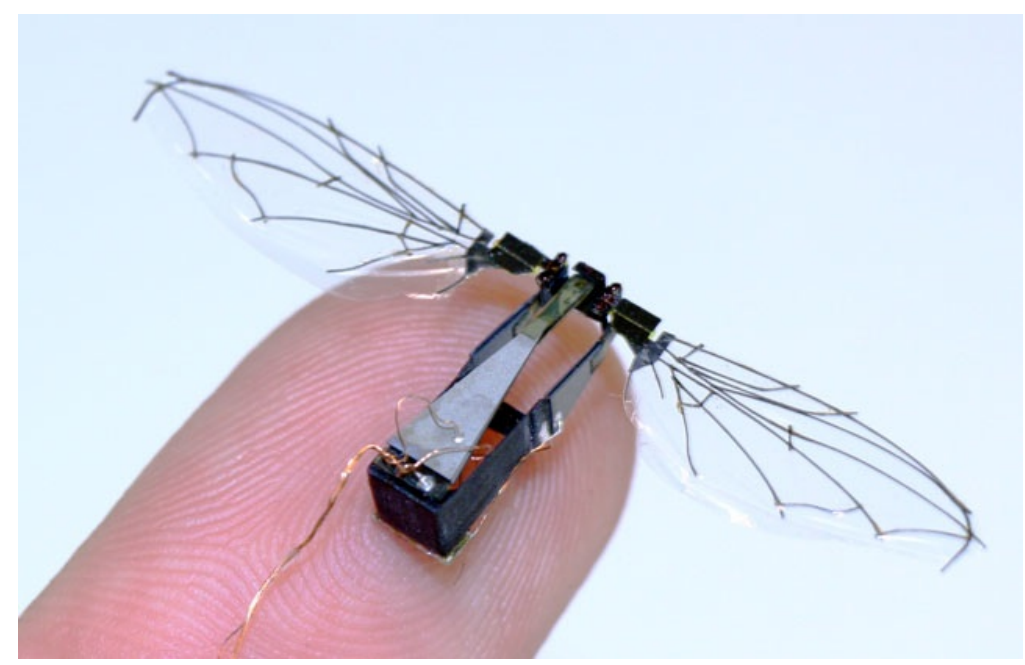

Figure 7. Next-generation Harvard microrobotic fly with biomimetic composite wings [10].

Current efforts seek an improved process that would enable rapid prototyping of wings; the process as described requires manual alignment and consequently is workersubjective. Possibilities include the use of laser-micromachined, isotropic materials 
for the veins, or filling the mold with shorter, milled carbon fibers and epoxy matrix. However, because both options would compromise the flexural stiffness and/or the anisotropy of the wing, efforts are ongoing in this area.

To enhance propulsive efficiency or control, the fabrication process could be expanded to include embedded micro-actuators into the wing. Active materials would be able to control deformation of individual wings, or more directly adjust the pressure distribution over the wing, as with the electrostatic valves in [35]. Insects do not use active musculature, but such innovations may prove to be superior to biological steering mechanisms.

Though we have investigated static bending here, the effect of flexural stiffness on dynamic properties is yet to be elucidated, as is the role of mass distribution. In the future, mass distribution will be quantified using 3D CAD software or a strip-weighing technique [13], and dynamic tests will be performed on fabricated wings attached to an actual transmission as in the Harvard Microbotic Fly (Fig. 7) and flapped through complex trajectories at biologically relevant frequencies in a horizontal stroke plane $[2,36]$. Sensors to measure the rapid, time-varying forces produced are under development [37]. This will allow us to correlate the measured forces with the corresponding instantaneous wing shape, as derived from spatial data captured by high speed videography. Such experiments will provide the basis for understanding the role of insect wing flexibility in force production, potentially leading to optimal rules for MAV wing design.

\section{Acknowledgments}

The authors thank Michael Petralia and the Harvard Center for Nanoscale Systems for assistance with fabrication. This work was supported in part by the Army Research Laboratory (award number W911NF-08-2-0004) and the National Science Foundation (award number CMMI-07466 38). Any opinions, findings and conclusions or recommendations expressed in this material are those of the authors and do not necessarily reflect those of the National Science Foundation.

\section{References}

[1] R. S. Fearing, K. H. Chiang, M. H. Dickinson, D. L. Pick, M. Sitti, and J. A. Yan, "Wing transmission for a micromechanical flying insect," in Proceedings. ICRA '00. IEEE International Conference on Robotics and Automation, 2000., K. H. Chiang, Ed., vol. 2, 2000, pp. 1509-1516 vol.2.

[2] R. Wood, "Design, fabrication, and analysis of a 3DOF, 3cm flapping-wing MAV," in IEEE/RSJ IROS, San Diego, CA, 2007.

[3] H. Tanaka, K. Hoshino, K. Matsumoto, and I. Shimoyama, "Flight dynamics of a butterfly-type ornithopter," in IEEE/RSJ IROS, 2005, pp. 2706-2711.

[4] T. Pornsin-Sirirak, Y. Tai, C. Ho, and M. Keennon, "Microbat: A palm-sized electrically 
powered ornithopter," Proceedings of NASA/JPL Workshop on Biomorphic Robotics, pp. 14-17, 2001.

[5] R. Madangopal, G. Student, Z. Khan, and S. Agrawal, "Biologically inspired design of small flapping wing air vehicles using four-bar mechanisms and quasi-steady aerodynamics," in Journal of Mechanical Design, vol. 127, 2005, p. 809.

[6] R. Dudley, The biomechanics of insect flight: form, function, evolution. Princeton, NJ: Princeton University Press, 2000.

[7] M. H. Dickinson, F.-O. Lehmann, and S. P. Sane, "Wing rotation and the aerodynamic basis of insect flight," Science, vol. 284, no. 5422, pp. 1954-1960, 1999.

[8] R. J. Wootton, "The mechanical design of insect wings," Scientific American, vol. 263, no. 5, pp. 114-120, 1990.

[9] A. M. Mountcastle and T. L. Daniel, "Wing stiffness affects mean advective flows of Manduca sexta, with wing overlap a potential contributor," in SICB, Boston, MA, 2009.

[10] B.M. Finio, J.K. Shang, and R.J. Wood, "Body torque modulation for a microrobotic fly," in IEEE ICRA, Kobe, Japan, 2009.

[11] A. R. Ennos, "The inertial cause of wing rotation in diptera," J Exp Biol, vol. 140, no. 1, pp. 161-169, 1988.

[12] R. Norberg, "The pterostigma of insect wings an inertial regulator of wing pitch," Journal of Comparative Physiology A: Neuroethology, Sensory, Neural, and Behavioral Physiology, vol. 81, no. 1, pp. 9-22, 1972.

[13] C. P. Ellington, "The aerodynamics of hovering insect flight. II. morphological parameters," Philosophical Transactions of the Royal Society of London. Series B, Biological Sciences, vol. 305, no. 1122, pp. 17-40, 1984.

[14] S. A. Combes and T. L. Daniel, "Shape, flapping and flexion: wing and fin design for forward flight," J Exp Biol, vol. 204, no. 12, pp. 2073-2085, 2001.

[15] M. Okamoto, K. Yasuda, and A. Azuma, "Aerodynamic characteristics of the wings and body of a dragonfly," J Exp Biol, vol. 199, no. 2, pp. 281-294, 1996.

[16] C. P. Ellington, "The aerodynamics of hovering insect flight. III. kinematics," Philosophical Transactions of the Royal Society of London. Series B, Biological Sciences, vol. 305, no. 1122, pp. 41-78, 1984.

[17] R. J. Wootton, "Leading edge section and asymmetric twisting in the wings of flying butterflies," J Exp Biol, vol. 180, pp. 105-117, 1993.

[18] A. R. Ennos, "Mechanical behaviour in torsion of insect wings, blades of grass and other cambered structures," Proceedings of the Royal Society of London, B, vol. 259, pp. 15-18, 1995.

[19] D. J. S. Newman and R. J. Wootton, "An approach to the mechanics of pleating in dragonfly wings," J Exp Biol, vol. 125, no. 1, pp. 361-372, 1986.

[20] A. B. Kesel, "Aerodynamic characteristics of dragonfly wing sections compared with technical aerofoils," J Exp Biol, vol. 203, no. 20, pp. 3125-3135, 2000.

[21] R. J. Wootton, "Functional morphology of insect wings," Annual Review of Entomology, vol. 37, no. 1, pp. 113-140, 1992.

[22] S. A. Combes and T. L. Daniel, "Flexural stiffness in insect wings I. scaling and the influence of wing venation," J Exp Biol, vol. 206, no. 17, pp. 2979-2987, 2003.

[23] — - "Flexural stiffness in insect wings II. spatial distribution and dynamic wing bending," $J$ Exp Biol, vol. 206, no. 17, pp. 2989-2997, 2003.

[24] C. P. Ellington, C. van den Berg, A. P. Willmott, and A. L. R. Thomas, "Leading-edge vortices in insect flight," Nature, vol. 384, no. 6610, pp. 626-630, 1996.

[25] R. J. Wootton, R. C. Herbert, P. G. Young, and K. E. Evans, "Approaches to the structural modelling of insect wings," Philosophical Transactions of the Royal Society of London B, vol. 358 , pp. $1577-1587,2003$. 
[26] S. P. Sane, "The aerodynamics of insect flight," J Exp Biol, vol. 206, pp. 4191-4208, 2003.

[27] H. Liu, C. P. Ellington, K. Kawachi, C. Van Den Berg, and W. A.P., "A computational fluid dynamic study of hawkmoth hovering," J Exp Biol, vol. 201, pp. 461-477, 1998.

[28] H. Aono, L. Fuyou, and H. Liu, "Near- and far-field aerodynamics in insect hovering flight: an integrated computational study," J Exp Biol, vol. 211, no. 2, pp. 239-257, 2008.

[29] W. Shyy, P. G. Ifju, and D. Vileru, "Membraned wing-based micro air vehicles", Appl. Mech. Rev., Vol. 58, No. 4, 283-302, Jul 2005.

[30] L. Zhao, Q. Huang, X. Deng, and S. Sane, "The effect of chord-wise flexibility on the aerodynamic force generation of flapping wings: experimental studies," in IEEE ICRA, Kobe, Japan, 2009.

[31] H. Tanaka, K. Matsumoto, and I. Shimoyama, "Fabrication of a three-dimensional insect-wing model by micromolding of thermosetting resin with a thin elastmeric mold," Journal of Micromechanics and Microengineering, no. 12, p. 2485, 2007.

[32] J.-H. Park, K.-J. Yoon, and H.-C. Park, "Development of bio-mimetic composite wing structures and experimental study on flapping characteristics," Robotics and Biomimetics, 200\%. ROBIO 200\%. IEEE International Conference on, pp. 25-30, 2007.

[33] R. Wood, S. Avadhanula, R. Sahai, and R. Fearing, "Microrobot design using fiber reinforced composites," J. of Mechanical Design, vol. 130, no. 5, 2008.

[34] Y. Xia and G. M. Whitesides, "Soft lithography," Annual Review of Materials Science, vol. 28, no. 1, pp. 153-184, 1998.

[35] M. Liger, N. Pronsin-Sirirak, Y-C. Tai, S. Ho, and C-H. Ho, "Large-area electrostatic-valved skins for adaptive flow control on ornithopter wings," Technical Digest, Solid State Sensor and Actuator Workshop (SSAW '02), pp. 247-250, Hilton Head Island, South Carolina, June 2002.

[36] R. Wood, "Liftoff of a 60mg flapping-wing mav," in IEEE/RSJ IROS, San Diego, CA, 2007.

[37] R. Wood, K.-J. Cho, and K. Hoffman, "A Novel Multi-Axis Force Sensor for Microrobotics Applications," in review J. Smart Materials and Structures, 2009. 